\title{
EXTREMAL COMPRESSIONS OF CLOSED OPERATORS
}

\author{
K.-H. FÖRSTER AND K. JAHN
}

(Communicated by Palle E. T. Jorgensen)

\begin{abstract}
Let $X$ be a Banach space, $A$ a closed linear operator on $X$, and $\lambda_{1}, \ldots, \lambda_{n}$ isolated eigenvalues of $A$ of finite multiplicity. If $P$ is a projection on $X$ such that $\lambda_{1}, \ldots, \lambda_{n}$ belong to the resolvent of the compression of $A$ on the range of $P$ it is easy to see that
\end{abstract}

$$
\operatorname{dim} N(P) \geq \max \left\{\operatorname{dim} N\left(\lambda_{i}-A\right): 1 \leq i \leq n\right\} .
$$

It is shown that there exist such projections where we have equality in this inequality.

Let $X$ be a complex Banach space. For an operator $A$ in $X$ (not necessarily everywhere defined) we denote by $D(A), N(A)$, and $R(A)$ the domain, the kernel, and the range of $A$, respectively. If $P$ is a bounded linear projection on $X$ with $P(D(A)) \subseteq D(A)$ the compression of $A$ on $R(P)$ is the linear operator $A_{P}$ defined in the closed subspace $R(P)$ by $D\left(A_{P}\right)=D(A) \cap R(P)$ and $A_{P} y=P A y$ for $y \in R(P)$. It is easy to see that $A_{P}$ is injective (i.e., $\left.N\left(A_{P}\right)=\{0\}\right)$ iff $N(A) \cap R(P)=\{0\}$ and $R(A P) \cap N(P)=\{0\}$. Therefore $\operatorname{dim} N(P) \geq \operatorname{dim} N(A)$ if $A_{P}$ is injective.

The collection of projections $P$ on $X$ with $P(D(A)) \subseteq D(A)$ will be denoted by $P_{A}(X)$. The main result of this note is the following:

Theorem. Let $A$ be a closed linear operator in a Banach space $X$ and let $\lambda_{1}, \ldots, \lambda_{n}$ be isolated eigenvalues of $A$ of finite (algebraic) multiplicity. Let $\Omega$ be a subset of $\mathbf{C}$ such that $\Omega \neq \mathbf{C}$ and $\Omega \cap \sigma(A)=\left\{\lambda_{1}, \ldots, \lambda_{n}\right\}$. If $\zeta \in \mathbf{C} \backslash \Omega$ then there is a projection $P \in P_{A}(X)$ such that $\Omega \subseteq \rho\left(A_{P}\right), \sigma\left(A_{P}\right)=$ $\sigma(A) \backslash \Omega \cup\{\zeta\}$ and

$$
\operatorname{dim} N(P)=\max _{\lambda \in \Omega} \operatorname{dim} N(\lambda-A) .
$$

In [1, 2] Islamov proved a similar result for finite-dimensional perturbations of $A$. It is well known that many results on compact or finite-dimensional perturbations have analogues in terms of compressions to subspaces of finite codimension, see $[5,6,7]$. The theorem above is another example of this relationship.

Received by the editors June 28, 1990.

1980 Mathematics Subject Classification (1985 Revision). Primary 47A20; Secondary 47A53, 47A55.

Key words and phrases. Compressions, closed linear operators. 
The proof will be divided into two steps. The main step is the following lemma which is the theorem in the finite-dimensional case. For its proof we need some facts about $\lambda$-matrices (for details see [4]).

A $\lambda$-matrix is a matrix over the ring of polynomials in $\lambda$ over $\mathbf{C}$. Let $B$ be an $n \times n$ matrix over $\mathbf{C}$ and $L_{B}(\lambda):=\lambda I-B$. For $l=1, \ldots, n$ define the polynomial $d_{l}(\lambda)$ to be the greatest common divisor ( gcd ) of all minors of $L_{B}(\lambda)$ of order $l$ and $d_{0}(\lambda) \equiv 1$.

$$
i_{l}(\lambda):=d_{l}(\lambda) / d_{l-1}(\lambda) \quad(l=1, \ldots, n)
$$

are called the invariant polynomials of $L_{B}(\lambda)$. Following holds (see $[4,4.10]$ ): Two $n \times n$ matrices $A, B$ are similar iff $L_{A}, L_{B}$ have the same invariant polynomials. Two $\lambda$-matrices $L_{1}(\lambda), L_{2}(\lambda)$ are said to be equivalent if there are $\lambda$-matrices $P(\lambda), Q(\lambda)$ with nonzero constant determinants such that $L_{1}(\lambda)=$ $P(\lambda) L_{2}(\lambda) Q(\lambda)$.

Lemma. Let $B$ be an $n \times n$ matrix and $\zeta \in \mathbf{C}$. There is a subspace $N$ of $\mathbf{C}^{n}$ and a projection $P$ onto $N$ such that for the compression $B_{P}$ holds $\sigma\left(B_{P}\right)=\{\zeta\}$ and

$$
\operatorname{dim} N(P)=\max _{\lambda \in \mathrm{C}} \operatorname{dim} N(\lambda-B) .
$$

Proof. Let $i_{r+1}(\lambda), \ldots, i_{n}(\lambda)$ be the nonconstant invariant polynomials of $L_{B}(\lambda)$. At first we show

$$
n-r=\max \{\operatorname{dim} N(\lambda-B): \lambda \in \mathbf{C}\} .
$$

It is well known (see e.g. $\left[4\right.$, p. 143, 148]) that $i_{l}(\lambda)$ divides $i_{l^{\prime}}(\lambda)$ for $l \leq l^{\prime}$. Thus for $\lambda_{0} \in \mathbf{C}$ we get the implications $i_{l}\left(\lambda_{0}\right)=0 \Rightarrow i_{k}\left(\lambda_{0}\right)=0$ for $l \leq$ $k \leq n$ and $i_{l}\left(\lambda_{0}\right) \neq 0 \Rightarrow i_{k}\left(\lambda_{0}\right) \neq 0$ for $1 \leq k \leq l$. By [4, Theorem 4.9.1] $L_{B}(\lambda)$ is equivalent to $\operatorname{diag}\left(i_{1}(\lambda), \ldots, i_{n}(\lambda)\right)$. So for every $\lambda_{0} \in \mathbf{C}$ we have $\operatorname{dim} N\left(\lambda_{0}-B\right)=\operatorname{dim} N\left(\operatorname{diag}\left(i_{1}\left(\lambda_{0}\right), \ldots, i_{n}\left(\lambda_{0}\right)\right)\right)=n-\min \left\{l: i_{1}\left(\lambda_{0}\right)=0\right\}+1$ (with $\min \{\}=n+1$ if $i_{n}\left(\lambda_{0}\right) \neq 0$ ). Taking the maximum on each side, we get $(*)$.

Now consider a polynomial $p(\lambda)=\lambda^{l}+\alpha_{l-1} \lambda^{l-1}+\cdots+\alpha_{0}(l>0)$ and its companion matrix

$$
L(p)=\left(\begin{array}{cccccc}
0 & 1 & 0 & \ddots & \cdots & 0 \\
\vdots & \ddots & \ddots & \ddots & \vdots \\
\vdots & & \ddots & \ddots & \ddots \\
0 & \cdots & \cdots & 0 & \ddots & 1 \\
-\alpha_{0} & \cdots & \cdots & -\alpha_{l-2}-\alpha_{l-1}
\end{array}\right)
$$

Considering invariant polynomials we next show that it is possible to choose suitable $\beta_{0}, \ldots, \beta_{l-1}$ such that $L(p)$ and

$$
C_{\zeta}(p)=\left(\begin{array}{cccccc}
\zeta & 1 & 0 & \cdots & \cdots & 0 \\
0 & \ddots & \ddots & \ddots & \\
\vdots & \ddots & \ddots & \ddots & \vdots \\
0 & \cdots & 0 & \ddots & \ddots & \ddots \\
\beta_{0} & \cdots & & \beta_{l-2} & \beta_{l-1}
\end{array}\right)
$$

are similar. 
By $[4,4.11] \lambda-L(p)$ has the invariant polynomials $1, \ldots, 1, p . \lambda-C_{\zeta}(p)$ has constant nonzero minors of order $1, \ldots, l-1$ independently of the choice of $\beta_{0}, \ldots, \beta_{l-1}$. So the first $l-1$ invariant polynomials are identically 1 . The last one is

$$
\operatorname{det}\left[\lambda-C_{\zeta}(p)\right]=(\lambda-\zeta)^{l}-\left(\beta_{l-1}-\zeta\right)(\lambda-\zeta)^{l-1}-\beta_{l-2}(\lambda-\zeta)^{l-2}-\cdots-\beta_{0}
$$

which induces the choice of $\beta_{0}, \ldots, \beta_{l-1}$ depending on $p$.

$B$ is similar to $\operatorname{diag}\left(L\left(i_{r+1}\right), \ldots, L\left(i_{n}\right)\right)[4,4.11 .1]$ and so similar to $D:=$ $\operatorname{diag}\left(C_{\zeta}\left(i_{r+1}\right), \ldots, C_{\zeta}\left(i_{n}\right)\right)$. Let $U$ be an invertible $n \times n$ matrix with $B=$ $U D U^{-1}$ and $l_{j}=\operatorname{deg} i_{j}(\lambda)$. Define $N_{1}$ to be the subspace of $\mathbf{C}^{n}$ spanned by the canonical vectors $e_{j}$ for $j \notin\left\{l_{r+1}, l_{r+1}+l_{r+2}, \ldots, l_{r+1}+l_{r+2}+\cdots+l_{n}\right\}$.

We set $N:=U N_{1}$ and $P=U P_{1} U^{-1}$, where $P_{1}$ denotes the orthogonal projection onto $N_{1}$. Then $P$ is a projection onto $N, \operatorname{dim} N(P)=\operatorname{dim} N\left(P_{1}\right)=$ $n-r . \quad B_{P}=V D_{P_{1}} V^{-1}$ where $V:=\left.U\right|_{N_{1}}: N_{1} \rightarrow N . B_{P}$ and $D_{P_{1}}$ have the same eigenvalues. $D_{P_{1}}$ is the matrix $D$ where rows and columns with indices $l_{r+1}, l_{r+1}+l_{r+2}, \ldots, l_{r+1}+\cdots+l_{n}$ are deleted, so $\sigma\left(B_{P}\right)=\{\zeta\}$. The lemma is proved.

Proof of the theorem. $\Omega \cap \sigma(A)=\left\{\lambda_{1}, \ldots, \lambda_{n}\right\}$ is a bounded part of $\sigma(A)$, separated from $\sigma(A) \backslash\left\{\lambda_{1}, \ldots, \lambda_{n}\right\}$. By [3, Theorem 6.17] we have a decomposition of $A$ according to a decomposition $X=M_{1} \oplus M_{2}$ in such a way that we have for $A_{P_{1}}, A_{P_{2}}\left(P_{1}\right.$ the projection onto $M_{1}$ along $\left.M_{2}, P_{2}=I-P_{1}\right)$ :

$$
\begin{gathered}
\operatorname{dim} P_{1} X=\operatorname{dim} M_{1}<\infty, \quad D\left(A_{P_{1}}\right)=M_{1} \\
\sigma\left(A_{P_{1}}\right)=\left\{\lambda_{1}, \ldots, \lambda_{n}\right\}, \sigma\left(A_{P_{2}}\right)=\sigma(A) \backslash\left\{\lambda_{1}, \ldots, \lambda_{n}\right\},
\end{gathered}
$$

and

$$
\operatorname{dim} N(\lambda-A)=\operatorname{dim} N\left(\lambda-A_{P_{1}}\right) \quad \text { for } \lambda \in \Omega \text {. }
$$

By the preceding lemma there is a subspace $N$ of $M_{1}$ and a projection $\widetilde{P}$ of $M_{1}$ onto $N$ such that $\operatorname{dim} M_{1} / N=\max \left\{\operatorname{dim} N\left(\lambda-A_{P_{1}}\right): \lambda \in \mathrm{C}\right\}$ and $\sigma\left(\left(A_{P_{1}}\right)_{P}=\{\zeta\}\right.$.

Set $M:=N \oplus M_{2}$ and $P:=\widetilde{P} P_{1}+P_{2}$. Then we have

$$
\begin{gathered}
\operatorname{codim}_{X} M=\operatorname{codim}_{M_{1}} N=\max _{\lambda \in \mathbf{C}} \operatorname{dim} N\left(\lambda-A_{P_{1}}\right)=\max _{\lambda \in \Omega} \operatorname{dim} N(\lambda-A), \\
P^{2}=P, \quad R(P)=M \quad \text { and } \quad \sigma\left(A_{P}\right)=\sigma(A) \backslash \Omega \cup\{\zeta\} .
\end{gathered}
$$

The proof is complete.

With this result it is possible to show a corollary for the Browder spectrum analogously to [7].

Corollary. Let $A$ be a bounded operator on a complex Banach space $X$ and $\varepsilon>0$. Then there is an extremal compression $A_{P}$ of $A$ in such a way that $\sigma\left(A_{P}\right)$ is contained in the $\varepsilon$-neighborhood $U$ of the Browder spectrum $\sigma_{b}(A)$ of $A$ and

$$
\operatorname{dim} N(P)=\max _{\lambda \notin U} \operatorname{dim} N(\lambda-A) .
$$

Proof. Consider $\sigma(A) \backslash U$, with $U=\left\{\lambda \in \mathbf{C}: \operatorname{dist}\left(\lambda, \sigma_{b}(A)\right)<\varepsilon\right\}$. This is a finite set because $\sigma(A) \backslash U$ is a compact set of isolated points. Applying the theorem to $A, \Omega:=\sigma(A) \backslash U$ and an arbitrary $\zeta \in \sigma_{\text {ess }}(A)$ shows the corollary. 


\section{REFERENCES}

1. G. G. Islamov, Controls of the spectrum of a dynamical system, Differential Equations $\mathbf{2 3}$ (1987), 872-875.

2. _ Extremal perturbations of closed operators, Izv. Vyssh. Uchebn. Zaved. Mat., no. 1 (1989), 35-41. (Russian)

3. T. Kato, Perturbation theory for linear operators, Springer-Verlag, Berlin and New York, 1976.

4. P. Lancaster, Theory of matrices, Academic Press, New York, 1969.

5. A. Pokrzywa, A characterization of the Weyl spectrum, Proc. Amer. Math. Soc. 92 (1984), 215-218.

6. J. Zemánek, Compressions and the Weyl-Browder spectra, Proc. Roy. Irish Acad. Sect. A. 86a (1986), 57-62.

7. _ Approximation of the Weyl spectrum, Proc. Roy. Irish Acad. Sect. A. 87a (1987), 177-180.

Technische Universität Berlin, Fachbereich Mathematik, Strasse des 17, Juni 136, 1000 Berlin 12, Federal Republic of Germany 